\title{
Metastasis of prostate cancer and melanoma cells in a preclinical in vivo mouse model is enhanced by L-plastin expression and phosphorylation
}

Selina M Riplinger ${ }^{1}$, Guido H Wabnitz ${ }^{1}$, Henning Kirchgessner ${ }^{1}$, Beate Jahraus ${ }^{1}$, Felix Lasitschka ${ }^{2}$, Bianca Schulte ${ }^{1}$, Gabri van der Pluijm ${ }^{3}$, Geertje van der Horst ${ }^{3}$, Günter J Hämmerling ${ }^{4}$, Inaam Nakchbandi ${ }^{1,5}$ and Yvonne Samstag ${ }^{1 *}$

\begin{abstract}
Background: Tumor cell migration and metastasis require dynamic rearrangements of the actin cytoskeleton. Interestingly, the F-actin cross-linking and stabilizing protein L-plastin, originally described as a leukocyte specific protein, is aberrantly expressed in several non-hematopoietic malignant tumors. Therefore, it has been discussed as a tumor marker. However, systematic in vivo analyses of the functional relevance of L-plastin for tumor cell metastasis were so far lacking.

Methods: We investigated the relevance of L-plastin expression and phosphorylation by ectopical expression of L-plastin in human melanoma cells (MV3) and knock-down of endogenous L-plastin in prostate cancer (PC3M). The growth and metastatic potential of tumor cells expressing no L-plastin, phosphorylatable or non-phosphorylatable L-plastin was analyzed in a preclinical mouse model after subcutaneous and intracardial injection of the tumor cells.

Results: Knock-down of endogenous L-plastin in human prostate carcinoma cells led to reduced tumor cell growth and metastasis. Vice versa, and in line with these findings, ectopic expression of L-plastin in L-plastin negative melanoma cells significantly increased the number of metastases. Strikingly, the metastasis promoting effect of L-plastin was not observed if a non-phosphorylatable L-plastin mutant was expressed.

Conclusions: Our data provide the first in vivo evidence that expression of L-plastin promotes tumor metastasis and, importantly, that this effect depends on an additionally required phosphorylation of L-plastin. In conclusion, these findings imply that for determining the importance of tumor-associated proteins like L-plastin a characterization of posttranslational modifications is indispensable.
\end{abstract}

Keywords: L-plastin, Actin cytoskeleton, Metastasis, Prostate cancer, Melanoma

\section{Background}

Development of metastasis causes the most serious clinical consequences of cancer and is responsible for over $90 \%$ of cancer related deaths [1]. The process of metastasis is complex. It involves release of tumor cells from the primary tumor, intravasation, dissemination within vessels, adhesion to vessel walls, extravasation as well as invasion and migration into the tissue of distant organs in order to colonize and form metastases. These steps require a high

\footnotetext{
* Correspondence: Yvonne.Samstag@urz.uni-heidelberg.de
${ }^{1}$ Institute for Immunology, Ruprecht-Karls-University, Heidelberg, Germany

* Correspondence: Yvonne.Samstag@urz.uni-heidelberg.de
${ }^{1}$ Institute for Immunology, Ruprecht-Karls-University, Heidelberg, Germany

Full list of author information is available at the end of the article
}

motility of cancer cells, which is mediated by modulation of the cellular cytoskeleton (for review see [2,3]). Recent studies indicate cytoskeleton binding proteins as important players in tumor metastasis [4,5] (for review see [6]), particularly by their capability to bind to and regulate integrin molecules [7]. Therefore, such molecules may be promising targets to inhibit the metastatic properties of tumor cells.

Interestingly, some cancers express the actin reorganizing protein L-plastin, which is normally leukocyte specific and not present in non-hematopoietic cells $[8,9]$. After the discovery of L-plastin as a tumor-associated protein, its 
role as a potential tumor marker was examined [10,11]. However, results of clinical studies comparing L-plastin expression in tumor specimen from patients with disease severity were ambiguous (for review see [12]). While expression of L-plastin correlated with the invasive potential of colorectal cancer [13], there was no correlation between L-plastin expression and tumor progression in breast cancer [14] or melanoma [15]. One reason for this discrepancy may be that these studies did not consider the phosphorylation status of L-plastin.

The plastin family of actin-binding proteins consists of three isoforms, which show tissue specific expression. They exhibit a similar molecular organization containing two consecutive actin binding domains in the C-terminus, each consisting of two calponin homology $(\mathrm{CH})$-domains. This structure allows the organization of actin filaments into very tight bundles. In the $\mathrm{N}$-terminal part there are two helix-loop-helix EF-hand $\mathrm{Ca}^{2+}$-binding motifs as well as a phosphorylation site on Ser5 (for review see [16]). Ser5 phosphorylation was shown to increase the actin bundling activity of L-plastin in vitro to promote its targeting to sites of actin assembly [17]. Regulation through phosphorylation of L-plastin has been described as a consequence of immune responses [18-20] as well as in response to signals triggering migration [21].

$\mathrm{L}$-plastin function is important for cells of the innate as well as the adaptive immune system. We have demonstrated that L-plastin is crucial for immune synapse formation [19]. Furthermore, it regulates integrin-dependent adhesion and migration of both granulocytes $[22,23]$ and T-cells [24]. From in vitro studies there were also hints that L-plastin plays a role in tumor cell motility (for review see $[12,25,26])$. However, so far no in vivo experiments existed investigating whether L-plastin plays a crucial role for tumor cell metastasis.

Therefore, in this study we systematically analyzed the in vivo role of L-plastin expression as well as L-plastin phosphorylation for tumor cell growth and tumor metastasis formation in a xenograft mouse model after subcutaneous or intracardial injection respectively of different human cancer cells.

\section{Results}

\section{Knock-down of L-plastin in human prostate cancer cells reduces tumor growth in vivo}

To investigate the effect of endogenous L-plastin expression on tumor cell behaviour, the L-plastin positive human prostate cancer cell line PC-3Mpro4/luc was used (in the following designated as PC3M). These PC3M cells, in addition, stably express luciferase and thus, exhibit luminescence in the presence of luciferin (Additional file 1: Figure S1A, upper two panels). L-plastin was phosphorylatable in these cells since PMA (phorbol 12-myristate 13acetate) stimulation of PC3M cells led to phosphorylation of the endogenous L-plastin, as detected by an antiphospho Ser5 L-plastin antibody (Figure 1A). To reduce L-plastin expression in PC3M cells we used a lentiviral system to deliver an L-plastin specific shRNA. In parallel a non-targeting shRNA was expressed. As illustrated in Figure 1B, treatment with L-plastin specific shRNA (LPL shRNA) resulted in an over $90 \%$ reduction in L-plastin protein expression in contrast to cells treated with nontargeting control shRNA (nt shRNA) or untreated PC3M (PC3M untreated). Note that bioluminescent activity was not influenced by shRNA transfection (Additional file 1: Figure S1A, lower two panels).

Contact dependent as well as anchorage independent cell growth was then analyzed in vitro. For contact dependent proliferation, cell growth on tissue culture plates was counted daily up to 96 hours (Figure 1C). The knock-down of L-plastin had no effect on proliferation in this system. Anchorage independent proliferation was determined with a soft agar assay [27]. This assay did also not unravel a growth disadvantage of PC3M cells due to a knock-down of L-plastin (Figure 1D). Together, knockdown of L-plastin had no effect on in vitro proliferation.

We next analyzed the in vivo tumor growth in a xenograft mouse model. PC3M cells either containing endogenous L-plastin, or PC3M cells expressing nt shRNA or the LPL shRNA were injected subcutaneously in the left leg of nude mice. These mice lack a thymus and are not able to induce an adaptive immune response against human cells [28]. Tumor growth was analyzed weekly over 42 days. Primary tumors were excised at day 42 and tumor volume was calculated. Surprisingly, knockdown of L-plastin reduced significantly the primary tumor growth in vivo (Figure $1 \mathrm{E}$ and $\mathrm{F}$ ). Since the in vitro proliferation was not significantly changed by knock-down of L-plastin, this diminished tumor growth could be due to a malfunction in colonialization.

\section{Knock-down of L-plastin interferes with processes crucial for colonialization of tumor cells}

In order to spread and colonize adjacent or non-adjacent tissues or organs, cancer cells need to migrate through the body. To investigate whether endogenous L-plastin expression in human tumor cells facilitates this process, we first analyzed the migratory potential of PC3M cells in vitro in transwell assays. Tumor cell metastasis is strongly influenced by stimuli, like chemokines or integrins, surrounding the tumor cells [29]. Since L-plastin promotes integrinmediated adhesion and migration of hematopoietic cells [16], we determined migration with the integrin ligand collagen I as a substrate and an additional chemoattractant (SDF1 $\alpha$ ) in the lower chamber of the transwell system (for details see Material and methods). Indeed, the knock-down of L-plastin in PC3M cells (PC3M LPL shRNA) significantly reduced migration (Figure 2 ). 


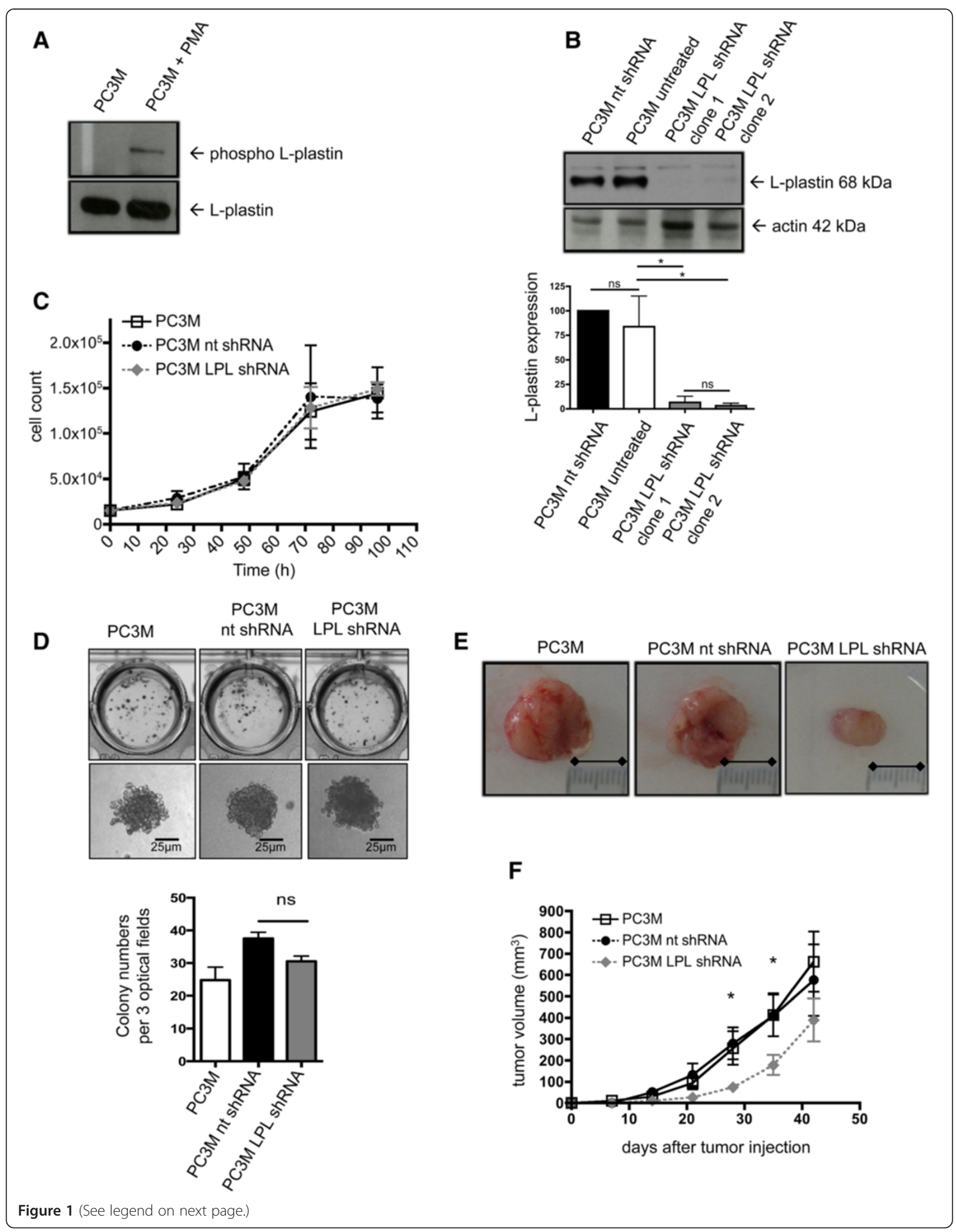


(See figure on previous page.)

Figure 1 L-plastin knock-down in prostate carcinoma cells. (A) L-plastin is expressed in PC3M cells and gets phosphorylated following PMA stimulation. Phosphorylation of L-plastin in untreated PC $3 \mathrm{M}$ or PC3M preincubated with $10^{-8}$ M PMA for 30 min was determined by Western blot with a phospho-Ser5 L-plastin specific antibody (upper panel). Total expression of L-plastin (lower panel). (B) ShRNA mediated downregulation of L-plastin in PC3M. PC3M infected with lentivirus encoding L-plastin (LPL) or non-targeting (nt) shRNA were analysed by Western blot for LPL protein (upper part), and $\beta$-actin as loading control. Quantification by densitometry of three independent experiments is shown in the lower part. For each experiment L-plastin levels were normalized for $\beta$-actin. The amount of L-plastin in untreated PC3M was set as $100 \%$. Results were analyzed using one-way ANOVA $(n=3)(*=p<0,05)$. (C) No influence of L-plastin knock-down on contact dependent in vitro proliferation of PC3M. Data represent means \pm SEM, experiments were repeated at least 3 times with samples in triplicate. (D) Unchanged anchorage independent in vitro proliferation of L-plastin knock-down PC3M. PC3M (as indicated) were allowed to grow in soft agar for 21 days. Cell culture plates with the colonies were scanned using a transmission light scanner (upper panel). Single colonies were acquired with a $20 x$ objective (lower panel). Bars indicate the colony numbers in three optical fields. Data represent means \pm SEM. Results were analyzed using one-way ANOVA ( $\mathrm{n}=4$; $\mathrm{ns}=$ not significant). (E/F) Knock-down of L-plastin in PC3M leads to reduced tumor volumes in vivo. PC3M (as indicated) were injected subcutaneously into mice. (E) Representative images of excised tumors at day 42. (F) Tumor volumes calculated weekly $\left(n=10\right.$; ${ }^{*} p<0.05$ for PC3M vs. PC3M LPL shRNA and PC3M nt shRNA vs. PC3M LPL shRNA).

\section{In vivo colonialization of human prostate cancer cells in a xenograft mouse model is reduced by knock-down of L-plastin}

The experiments shown in Figure 2 suggest a function of L-plastin in the process of cancer cell spreading and induction of metastatic colonies. In the so far here used experimental model of subcutaneous tumor cell injection no spontaneous metastasis to non-adjacent organs was detected. Therefore, we switched to an established metastasis model involving intracardial injection of tumor cells in nude mice [30], to analyze whether L-plastin promotes tumor metastasis. After injection of either PC3M cells containing endogenous L-plastin or L-plastin knock-down PC3M cells (PC3 LPL shRNA) into the left cardiac ventricle of nude mice, the number of metastatic colonies was assessed by weekly ventral and dorsal bioluminescence imaging of mice and counting of luciferase-positive foci from both sides. Figure 3A shows dorsal images of day 7, 21 and 42. Indeed, L-plastin knock-down PC3M cells showed significantly reduced numbers of metastatic colonies in the animals over a period of 42 days compared to animals injected with nt shRNA transfected PC3M or untransfected PC3M cells (Figure 3A and B). These data demonstrate that L-plastin expression promotes tumor metastasis by enhancing colonialization.

\section{Ectopically expressed wildtype L-plastin undergoes} spontaneous phosphorylation on Ser 5 in melanoma cells For an independent confirmation that L-plastin plays a role in in vivo colonialization of tumor cells the human melanoma cell line MV3 was used, since these cells were negative for endogenous L-plastin expression. This gives the possibility to analyze the effect of L-plastin on metastasis even in a phosphorylation-dependent manner via a transfection of wildtype or non-phosphorylatable L-plastin into these melanoma cells. To allow in vivo imaging of tumor cells, first, a cDNA encoding the luciferase 2 gene (pCAGG3.1-luc2) was transfected in MV3 cells and luciferase 2 expressing cells (designated as MV3) were selected, cloned and tested for their bioluminescence activity as well as for their proliferative and migratory capability. Luciferase expression had no influence on proliferation and migration of the cells (data not shown). Cells with a stable and strong bioluminescence activity (Figure 4A) were chosen for further transfections to generate stable L-plastin expressing clones.

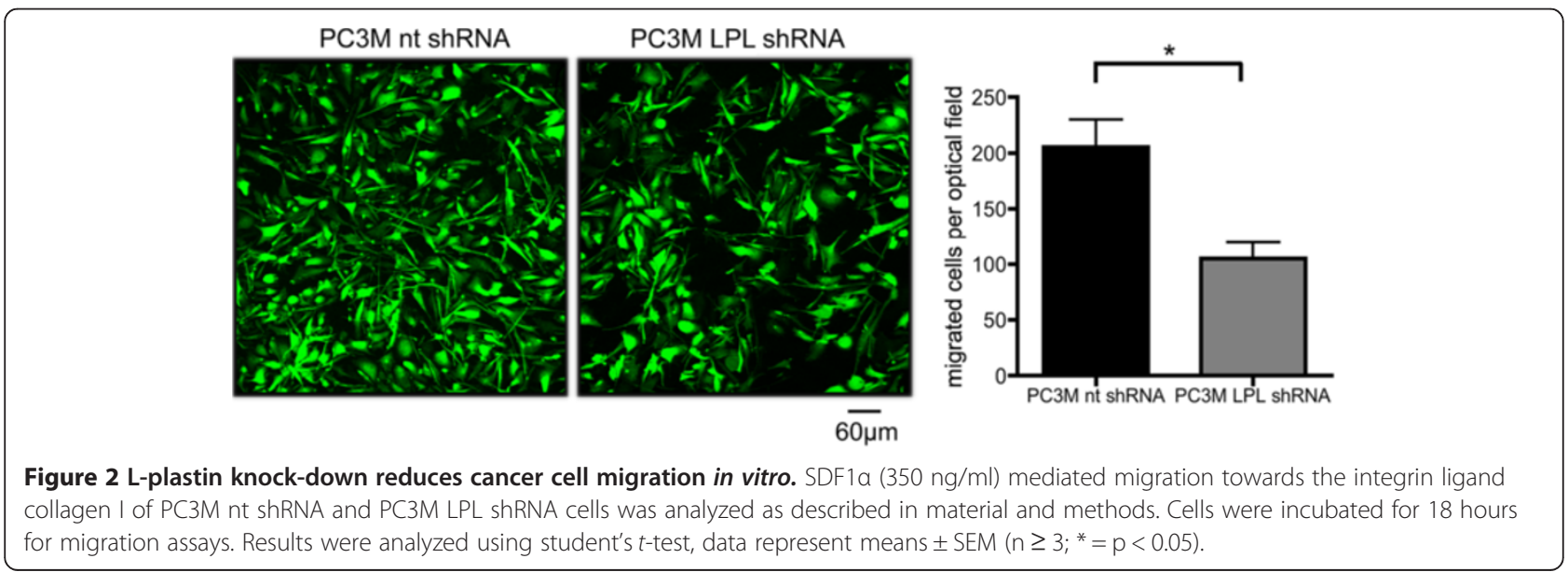



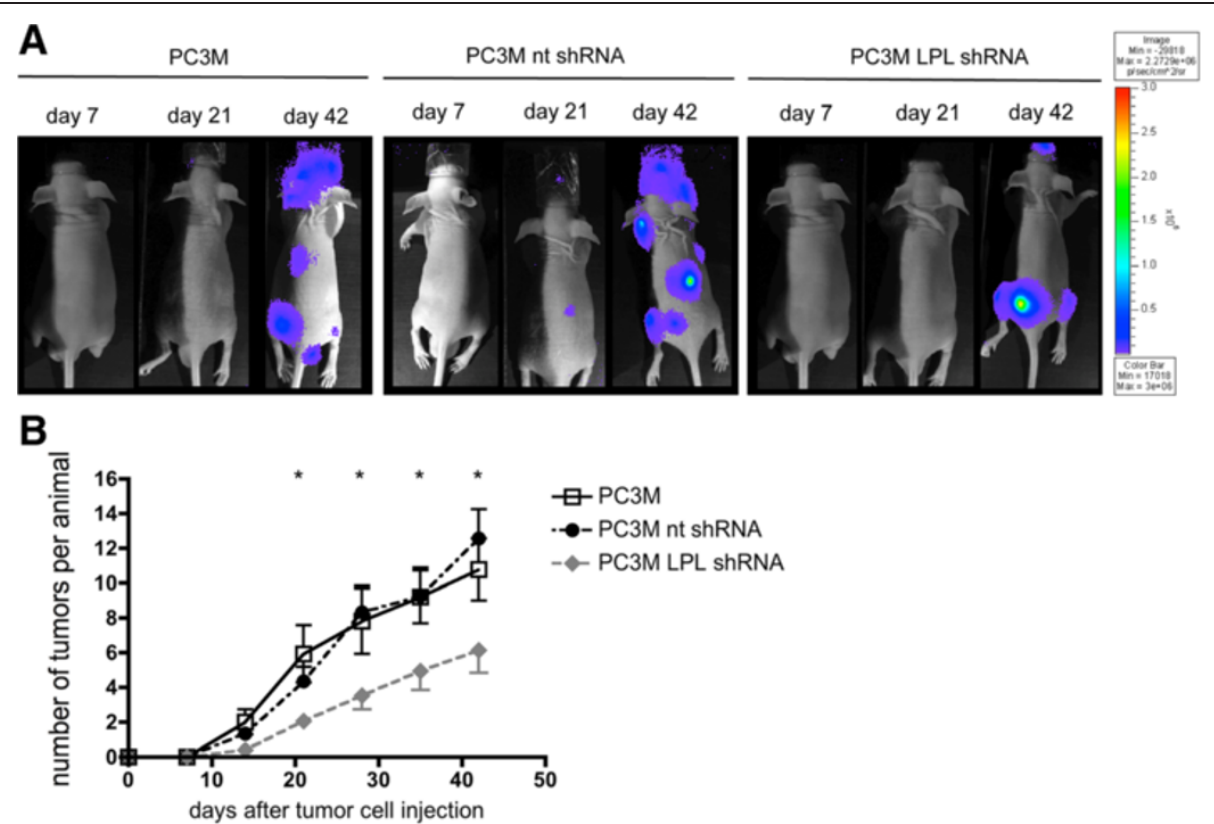

Figure 3 L-plastin knock-down reduces metastasis of prostate carcinoma cells. Tumor metastasis monitored by in vivo bioluminescence imaging. (A) Representative examples of dorsal optical images of mice injected with PC3M, PC3M nt shRNA or PC3M LPL shRNA cells at the indicated time points are shown. (B) Quantification of metastasis. Numbers of single spots, representing metastatic lesions, were counted from a ventral and dorsal view per animal and plotted over time after cancer cell injection. Results were analyzed using student's t-test, data represent means \pm SEM ( $n=12$ for PC3M and PC3M nt shRNA; $n=17$ for PC3M LPL shRNA; ${ }^{*}=p<0.05$ for PC3M vs. PC3M LPL shRNA and PC3M nt shRNA vs. PC3M LPL ShRNA).

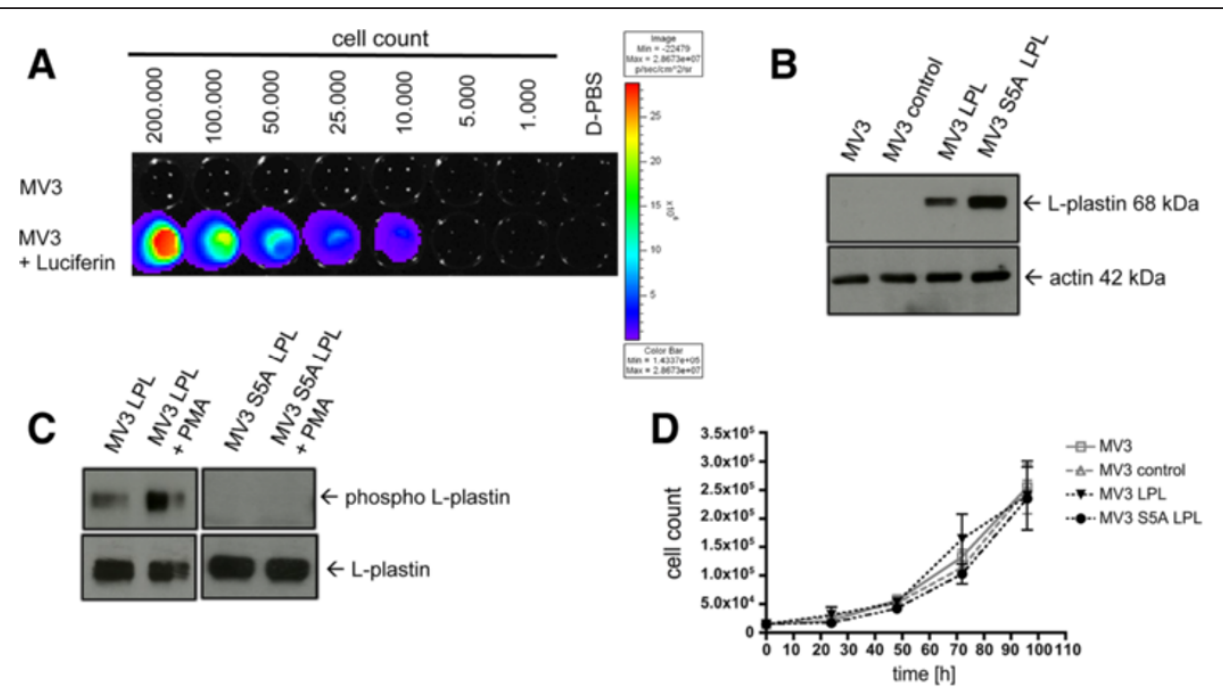

Figure 4 Phosphorylation of L-plastin in melanoma cells occurs spontaneously at Ser5. (A) Bioluminescence imaging of MV3 pCAGG3.1luc2 clone 2 (MV3) cells in vitro. The color bar on the right indicates the signal intensity range $\left(\mathrm{p} / \mathrm{s} / \mathrm{cm}^{2} / \mathrm{sr}\right.$ ). (B) Western blot analysis of L-plastin levels in different MV3 cells: untransfected MV3 cells (MV3), MV3 cells transfected with a control vector (MV3 control), or MV3 cells expressing either LPL (MV3 LPL) or S5A LPL (MV3 S5A LPL). (C) L-plastin phosphorylation, detected via a phospho Ser5 L-plastin antibody in Western Blot, occurs in MV3 cells expressing WT LPL plus and minus PMA stimulation but not in MV3 cells expressing S5A LPL. (D) Proliferation of the different MV3 cells in vitro. Data represent means \pm SEM, experiments were repeated at least three times with samples in triplicates. 
Up to now, the exact phosphorylation site of L-plastin in melanoma cells was not determined. From our previous work we know, that a S5AS7A mutant of L-plastin prevents phosphorylation [15]. In leukocytes and Vero cells Ser5 was identified as the site of phosphorylation $[17,18,22,31]$. To investigate if Ser5 is also the crucial site of phosphorylation in melanoma cells, a cDNA encoding wildtype L-plastin (LPL) or a mutated version of L-plastin (S5A LPL) were transfected into MV3 cells. Immunoblot analysis of generated cell clones revealed no expression of L-plastin in untransfected cells or cells transfected with a vector control (MV3 control), but high L-plastin expression was found in MV3 LPL and MV3 S5A LPL cells (Figure 4B). Note that the bioluminescent activity was not influenced by transfection or L-plastin expression (Additional file 1: Figure S1B). Interestingly, the wildtype L-plastin is already partially phosphorylated in the absence of further external stimuli. After stimulation of these cells with the phorbol ester PMA, the amount of phosphorylated L-plastin was enhanced as observed for the PC3 cells. These findings, in addition, imply that a protein kinase $\mathrm{C}$ is involved in the phosphorylation of L-plastin in these tumor cells. Such an L-plastin phosphorylation was, however, only observed in wildtype L-plastin expressing cells. In contrast, S5A mutation within the L-plastin molecule prevented phosphorylation as detected with a specific anti phospho Ser5 L-plastin antibody (Figure 4C) or in an assay analyzing total L-plastin phosphorylation (Additional file 2: Figure S2). Together, these data suggest that Ser5 is the functional relevant phosphorylation site of L-plastin for melanoma cell colonialization in vivo. In line with the data obtained with PC3M cells, expression of L-plastin had no effect on tumor cell proliferation in vitro (Figure 4D).

\section{L-plastin phosphorylation on Ser5 is required for the promoting effect of L-plastin on tumor cell migration in vitro}

Following the biochemical proof that mutation at Ser5 to alanine prevents phosphorylation, we next checked the functional consequences of L-plastin phosphorylation on Ser5 in melanoma cells. Thus, MV3 cells expressing either wildtype or S5A L-plastin were tested for their migratory and invasive potential in vitro. With collagen I as substrate (Figure 5A) transwell assays showed increased migration in the presence of wildtype L-plastin expression (MV3 LPL). This increase in the migratory capacity was abolished, if MV3 cells expressed only the non-phosphorylatable S5A LPL (Figure 5A). Chemotaxis in the absence of adhesive substrates was not influenced by either expression or phosphorylation of L-plastin, indicating that phosphorylation of Ser5 in L-plastin is important for integrin-mediated

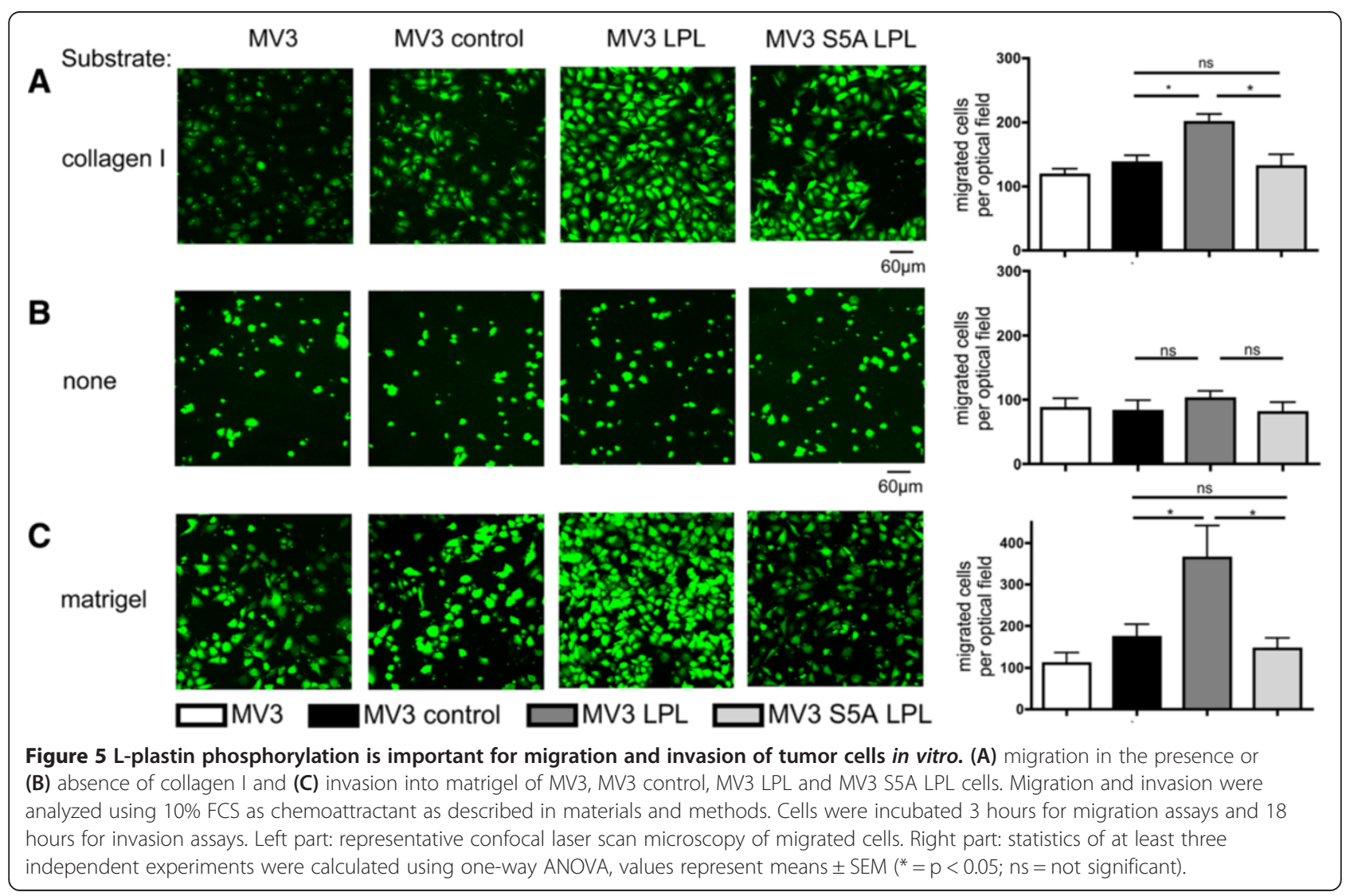


migration (Figure 5B). In a next step, invasiveness was investigated using a matrigel invasion assay [15]. Again, expression of L-plastin increased the number of transmigrating cells in contrast to cells without L-plastin expression or cells expressing the S5A LPL (Figure 5C). Thus, only expression of L-plastin phosphorylatable on Ser5 facilitated substrate-guided migration and invasiveness of MV3 cells in vitro.

\section{L-plastin enhances in vivo metastasis of human} melanoma cells in a phosphorylation dependent manner The subsequent experiments were designed to determine whether expression of L-plastin and its phosphorylation on Ser5 are crucial not only for melanoma cell migration and invasion in vitro, but also for the enhancement of tumor cell metastasis in vivo. Indeed, expression of wildtype L-plastin in MV3 cells led to an increased number of metastatic colonies in mice after intracardial injection of the tumor cells (Figure 6A). In marked contrast, expression of the non-phosphorylatable L-plastin variant did not influence cancer cell metastasis. Figure 6B shows representative bioluminescence images on day 5, 13, 27 from all groups. To investigate whether wildtype L-plastin is still phosphorylated in the metastatic colonies in vivo, tumors were excised and analyzed by immunohistochemistry using the phospho L-plastin antibody. Figure 6C demonstrates that phosphorylated L-plastin can be detected in metastatic tumor cells. Together, these data demonstrate, that ectopic expression of L-plastin in tumor cells enhances metastasis and that, strikingly, not only expression, but also phosphorylation of L-plastin is important to promote tumor metastasis.

\section{Discussion}

In order to metastasize, tumor cells must be able to leave the primary tumor and to colonize other tissues.

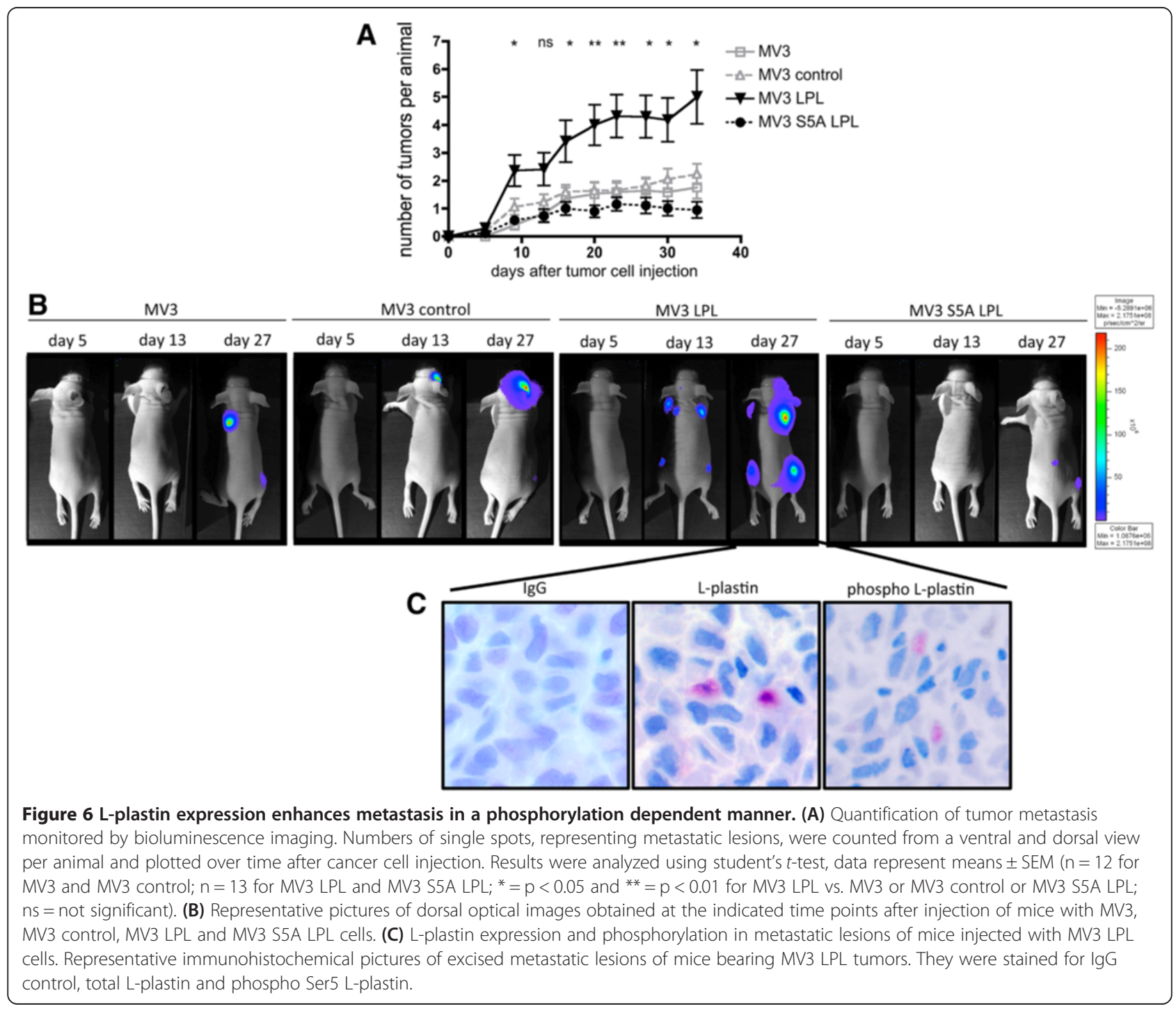


One important step in this process is the dynamic rearrangement of the actin cytoskeleton via actin binding proteins (for review see [6]). In contrast to untransformed tissue cells, a variety of human tumor cells express the primarily leukocyte specific protein L-plastin [9]. Yet, its cellular function in vivo and its causal relationship to tumor cell metastasis remained unclear. Thus, contradictory results regarding the correlation of L-plastin expression with tumor progression in patients in different tumor entities were found [13-15].

Hints for a role of L-plastin expression and its phosphorylation in metastasis came from our prior experiments showing that expression of wildtype L-plastin, but not of its non-phosphorylatable counterpart S5AS7A L-plastin strongly enhanced the capacity of MV3 melanoma cells to invade into matrigel in vitro [15]. These studies gave rise to the assumption that (i) the expression of L-plastin and (ii) its ability to become phosphorylated may provide tumor cells an advantage to form metastases. Our aim was to test this hypothesis for human cancer cells in vivo by using a xenograft mouse model. We found that expression of L-plastin in tumor cells indeed promotes their metastasis in mice. This could be demonstrated in two independent experimental systems, namely either knock-down of endogenous L-plastin expression in human prostate carcinoma cells or cDNA mediated L-plastin expression in L-plastin negative melanoma cells and subsequent injection of these cells into mice. Tumor cell dissemination and metastatic colony formation was followed by bioluminescence imaging and was finally confirmed by histological analysis. Metastases occurred preferentially in bone, which is likely in part due to the injection of the tumor cells into the left ventricle of the heart resulting in arterial distribution. ShRNA induced lower expression levels of endogenous L-plastin resulted in decreased metastasis. In line with this finding ectopic expression of wildtype L-plastin raised the amount of metastatic spots in the mice. Importantly, in contrast to wildtype L-plastin, expression of non-phosphorylatable L-plastin (S5A LPL) in melanoma cells did not increase their metastatic potential. These analyses demonstrate for the first time in vivo that L-plastin expression combined with L-plastin phosphorylation on Ser5 promotes tumor metastasis, suggesting that this combination may be an important risk factor for cancer patients. Coming along with a higher number of metastatic colonies due to L-plastin expression and phosphorylation, a trend towards a prolonged survival of the animals was observed if L-plastin was not expressed, or if the non-phosphorylatable mutant (S5A LPL) was expressed in the tumor cells (data not shown).

Not just the formation of metastatic colonies after intracardial injection of tumor cells in mice was reduced, but also the primary subcutaneous tumor growth was inhibited. Considering no influence of L-plastin neither on contact dependent nor on anchorage independent proliferation of the tumor cells in vitro, further aspects of the process of the outgrowth of a primary tumor seem to be influenced, namely the adhesion and colonialization of the tumor cells after injection. Adhesion, which is next to the migration of tumor cells one of the main steps of colonialization, is coordinated by integrins. Mechanistically, the enhanced metastatic behaviour of wildtype L-plastin expressing cancer cells may be also mediated by avidity regulation of integrins, since only migration in the presence of integrin ligands was enhanced by expression of wildtype L-plastin. In contrast, expression of non-phosphorylatable L-plastin did not show this effect. The resulting hypothesis that phosphorylation of L-plastin is crucial for its action on integrins is supported by the fact that L-plastin-derived peptides induced activation of $\alpha v \beta 3$-mediated adhesion of erythroleukemia cells [23] as well as by recent data showing that L-plastin expression [19] and phosphorylation [20,32] regulates the accumulation of the $\beta 2$-integrin LFA- 1 at the immune synapse of T-cells and antigen-presenting cells. A direct interaction of L-plastin with $\beta$-integrins was demonstrated for T-cells $[19,20]$ as well as for breast (MCF-7) or prostate (PC3) cancer cells [33]. Notably, in vitro, L-plastin was found in focal adhesion sites of MV3 melanoma [15] and Vero kidney epithelial cells [34]. For the latter it was even demonstrated that L-plastin phosphorylation modulates actin dynamics in focal adhesions. Thus, L-plastin phosphorylation may be important for integrin-mediated tumor cell adhesion: Firstly, via connecting integrins to the actin cytoskeleton and thereby increasing the integrin avidity at podosomes. Secondly, via modulating the actin cytoskeleton within these adhesive structures, which is important for stabilizing these complexes. Interestingly, the activity of matrix degrading enzymes, like seprase, is also regulated by integrin clustering within invadopodia [35]. Thus, a connection between L-plastin phosphorylation and integrin avidity regulation may explain the enhanced metastatic behaviour of L-plastin expressing tumor cells observed in vivo, since integrins play an important role for tumor cell migration, extravasation and adhesion. The identification of the integrins involved in L-plastin mediated tumor metastasis will be the subject of further investigations. Note, however, that the behaviour of cancer cells in vivo is strongly influenced by the local microenvironment. Thus, the dependence of a certain tumor on L-plastin expression and phosphorylation as well as its mode of function can vary in different tumor entities.

Taken together, our systematic in vivo analysis demonstrates for the first time that ectopic expression of L-plastin promotes tumor metastasis. Importantly, this L-plastin needs to be phosphorylatable on Ser5. Failure to take this L-plastin phosphorylation into account in studies, aiming to find a correlation between L-plastin 
expression and the severity of cancer diseases, may explain the non-conclusive results found in different tumor entities of patients [13-15]. Thus, future studies are needed to establish whether L-plastin phosphorylation on Ser5 can serve as a prognostic marker. Finally, our results suggest tissue or tumor specific inhibition of L-plastin phosphorylation as a potential new therapeutic strategy to prevent metastasis of cancer. In this regard L-plastin has the advantage that, unlike other actin-binding proteins, it is not expressed in untransformed cells of the respective non-hematopoietic tissues.

\section{Conclusion}

In summary, we show for the first time in vivo, via a preclinical mouse model, that ectopic expression of L-plastin in tumor cells promotes metastasis. Importantly, not the expression of L-plastin alone, but rather its additionally required phosphorylation on Ser5, enhances metastasis. Therefore, L-plastin phosphorylation on Ser5 may serve as novel prognostic marker. Given that L-plastin is selectively expressed in malignant cells - but not in untransformed non-hematopoietic cells - targeting of L-plastin expression or inhibition of L-plastin phosphorylation may open up novel therapeutic strategies to prevent tumor metastasis.

\section{Materials and methods \\ Cell culture}

The human prostate carcinoma cell line PC-3Mpro4/luc (in the following abbreviated as PC3M) (a generous gift of Dr. M. Cecchini, University of Berne, Switzerland) [36] was grown in high glucose DMEM medium supplemented with $10 \%$ fetal calf serum (FCS), w/o L-glutamine. The human melanoma cell line MV3 (a generous gift of Dr. van Muijen, University Hospital Nijmegen, The Netherlands) was grown in RPMI 1640 medium supplemented with $10 \%$ FCS, $2 \mathrm{mM}$ glutamine at $37^{\circ} \mathrm{C}$ in an humidified atmosphere containing $5 \% \mathrm{CO}_{2}$. Cell lines were tested by genome sequencing (DSMZ, Braunschweig, Germany).

\section{Generation of an anti phospho Ser5 L-plastin antiserum}

Rabbit polyclonal anti-phospho L-plastin antiserum against Ser5 phosphorylated L-plastin was produced by Eurogentec according to the Speedy 28-day program. This antiserum was generated against an L-plastin peptide in which Ser5 was phosphorylated. Western blotting with the purified antiserum was performed in a dilution of 1:2000.

\section{Mice}

BALB/c nude Mice (CAnN.Cg-Foxn $1^{n u} / \mathrm{Crl}$ ) were purchased from Charles River and were maintained in a specific pathogen-free facility of the Heidelberg University with controlled light/dark rhythm, temperature and humidity in concordance with animal care regulations.
Cages, bedding, food and water were all autoclaved. All experimental procedures involving animals were approved by the responsible agency in compliance with institutional and German governmental requirements.

\section{Lentivirus-mediated knock-down and generation of stable} knock-down cells

MISSION $^{\circ}$ Lentiviral Transduction Particles, containing short hairpin (sh) RNA lentiviral vectors based on pLKO.1puro containing a puromycin resistance gene and targeting five different sequences on the human L-plastin gene and a non-targeting shRNA vector as a control, were obtained from Sigma-Aldrich (Munich). Self-inactivating replication incompetent viral particles containing shRNAs were obtained from ImaGenes (Berlin). Infection was performed according to the manufacturer's instructions. The shRNA used included the mature sense sequence for L-plastin: CCGGGCGGACATTTAGGAACTGGATCTCGAGAT CCAGTTCCTAAATGTCCGCTTTTTG.

\section{Plasmids}

The cDNA of wild-type (wt) full-length L-plastin and S5A-L-plastin, which were used to clone the expression plasmid vectors, were described before [15]. The entire coding region of wt full-length L-plastin CDNA (LPL) and S5A-L-plastin (S5A LPL) cDNA were amplified from Lplastin or S5A L-plastin by polymerase chain reaction (PCR) introducing a 5' and 3'BamHI restriction site and inserted into $B a m H I$ digested pSELECT-hygro-mcs vector (InvivoGen). pSELECT-hygro-mcs with an EGFP insert served as control.

The pCAGG3.1-luc2 plasmid containing the mammaliancodon optimized firefly luciferase 2 was used as described elsewhere [37]. All constructs were checked by standard DNA sequencing.

\section{Transfections and establishment of stable protein expression in cell populations}

Plasmids expressing the firefly luciferase gene (pCAGG3.1luc2) and plasmids expressing either wt- or S5A-L-plastin cDNA or EGFP were transfected into MV3 cells using Lipofectamine 2000 reagent (Invitrogen) according to the manufacturer's instructions. Stable expression of transferred luciferase gene was controlled by bioluminescence imaging in complete medium supplemented with $150 \mu \mathrm{g} / \mathrm{ml} \mathrm{D}$-luciferin (Synchem) by in vitro imaging using the IVIS ${ }^{\text {max }}$ camera system (Xenogen, Alameda, CA, USA). Bioluminescent single cell clones were amplified in culture and tested for stable luminescence and proliferative potential in vitro.

Stable expression of transferred L-plastin genes was checked by immunoblots. L-plastin expressing single cell clones were amplified in culture and characterized for stable luminescence levels, L-plastin expression as well as 
migration in vitro. Positive MV3 LPL or MV3 S5A LPL clones, respectively, were expanded and initially selected and used for further in vitro and in vivo studies.

\section{SDS-Polyacrylamide gel electrophoresis followed by Western-blotting}

Protein lysates from cells were obtained as previously described [15]. Cells were washed once in ice-cold Trisbuffered saline (TBS), lysed for 30 minutes in TKMbuffer (50 mM Tris-HCl pH 7.5, 1\% NP40, $25 \mathrm{mM} \mathrm{KCl,}$ $5 \mathrm{mM} \mathrm{MgCl}_{2}, 1 \mathrm{mM} \mathrm{NaVO}_{4}, 5 \mathrm{mM} \mathrm{NaF}, 20 \mu \mathrm{g} / \mathrm{ml}$ each Leupeptin/Aprotinin) and nuclei were removed by centrifugation. SDS-polyacrylamide gel electrophoresis (SDSPAGE) was performed according to standard procedures. Proteins were blotted onto polyvinylidene difluoride (PVDF, Immobilon-P, Merck-Millipore, Darmstadt, Germany) membranes. Membranes were blocked with 10\% BSA in PBS. For protein detection the membranes were incubated with primary antibodies for L-plastin (clone LPL4A.1, Neomarkers) (1 $\mu \mathrm{g} / \mathrm{ml}$ ), phospho L-plastin (our own antibody) or 3 -actin (clone A2066, Sigma-Aldrich) (1:900) overnight at $4^{\circ} \mathrm{C}$, followed by incubation with horseradish peroxidase-conjugated anti-rabbit or anti-mouse secondary antibodies (Jackson ImmunoResearch). Proteins were visualized with ECL (Amersham-Pharmacia). Densitometric analysis was performed using a GS-800-densitometer and "Quantitiy One" software (BioRad). L-plastin phosphorylation was calculated as the ratio between phosphoprotein and total protein. Data from densitometric analysis are reported as mean \pm SEM.

\section{In vitro cell proliferation and soft agar assays}

For contact dependent proliferation, cells were washed, trypsinized and adjusted to a density of $1.5 \times 10^{4}$ cells $/ \mathrm{ml}$ in culture medium. Cells in $1 \mathrm{ml}$ aliquots were seeded to grow in 24-well plastic plates. Cell numbers were counted at the times indicated using a Neubauer chamber.

The soft agar assays (anchorage independent proliferation) were performed by preparing a $0.5 \%$ base agar and a $0.35 \%$ top agar with RPMI1640 and FCS (10\% v/v final concentration). The layer of $0.5 \%$ agar was poured in 24 well plates. PC3M, PC3M nt shRNA or PC3M LPL shRNA cells were resuspended in $0.35 \%$ top agar at a density of $1.5 \times 10^{5}$ cells $/ \mathrm{ml}$. Cell suspensions were poured on the top of the base layer. After solidification the agar was overlaid with RPMI1640 plus 10\% FCS and plates were incubated at $37^{\circ} \mathrm{C}$ in the presence of $5 \%$ $\mathrm{CO}_{2}$. The colonies were counted in 3 optical fields in each sample at day 13 .

\section{In vitro migration assay}

In vitro xcell migration was analyzed essentially as previously described [15], using transwell migration inserts (PET membrane, $8 \mu \mathrm{m}$ pore size, $6 \mathrm{~mm}$ diameter, BD
Biosciences) coated with $20 \mu \mathrm{g} / \mathrm{ml}$ collagen I in $0.02 \mathrm{~N}$ acetic acid at the bottom side and containing soluble chemoattractants $(600 \mu \mathrm{l}$ of either RPMI 1640 medium containing SDF1 $\alpha$ (350 ng/ml) for PC3M cells, or RPMI 1640 supplemented with 10\% FCS for MV3 cells) in the lower compartment. Cells were labeled for $15 \mathrm{mi}$ nutes at $37^{\circ} \mathrm{C} / 5 \% \mathrm{CO}_{2}$ with $1 \mu \mathrm{M}$ CFDA-SE (molecular probes) in PBS prior to performing the migration assay. $2.5 \times 10^{5}$ cells $/ \mathrm{ml}$ for MV3 and $1.5 \times 10^{5}$ cells $/ \mathrm{ml}$ for PC3M were allowed to transmigrate, then filters were removed and cells from the upper membrane surface were wiped off with a cotton swab. Filters were then washed, fixed and mounted on glass slides. Cells that had migrated to the coated lower side of the filter were detected by confocal laser scan microscopy and cells in four defined optical fields were counted for each filter. Time points are indicated in the figure legends.

\section{In vitro invasion assay}

To investigate the invasiveness of tumor cells BD BioCoat Matrigel invasion chambers were used. $200 \mu \mathrm{l}$ of a cell suspension at a concentration of $1 \times 10^{5}$ cells $/ \mathrm{ml}$ was added to the upper compartment and cells were allowed to migrate to the lower compartment with $10 \%$ of FCS as a chemoattractant. Invasive cells were counted as mentioned above.

\section{Subcutaneous injection of tumor cells}

5 week old male BALB/c nude mice were subcutaneously injected with $1.5 \times 10^{5}$ PC3M cells in $100 \mu \mathrm{l}$ D-PBS in the hind leg. Subcutaneous tumor growth was determined weekly via caliper. Tumor volume was calculated by the formula: length $\times$ width $^{2} \times 0.5$.

\section{Intracardial injection of tumor cells}

5 week old nude mice were anesthetized by intraperitoneal injection of $120 \mathrm{mg} / \mathrm{kg}$ ketamine hydrochloride with $16 \mathrm{mg} / \mathrm{kg}$ xylazine on the day of injection. Male animals were used for the injection of PC3M cells, female mice were used for the injection of MV3 cells. On day 0 , anesthetized animals were injected with $1.5 \times 10^{5}$ PC3M cells, or $1 \times 10^{5}$ MV3 cells respectively, diluted in $100 \mu \mathrm{l}$ D-PBS into the left cardiac ventricle. Anesthetized animals were placed in the IVIS100 Imaging System (Xenogen, Alameda, CA, USA) within 60 minutes after injection and imaged dorsally and ventrally $5 \mathrm{~min}$ after intraperitoneal injection of D-luciferin. A successful intracardial injection was indicated on day 0 by images showing systemic bioluminescence distributed throughout the body of the mouse. Only animals with evidence of a satisfactory injection continued the experiment. Assessment of subsequent metastasis was monitored in vivo once or twice a week, depending on the tumor model, for up to 42 days. 


\section{Bioluminescence imaging}

Bioluminescence imaging was performed with a CCD camera in a light-tight specimen box (IVIS100, Xenogen). Quantification of signals was performed by the acquisition and analysis software Living Image ${ }^{\bullet}$ (Xenogen). For in vitro imaging, bioluminescent cells were serially diluted from $1 \times 10^{3}$ to $2 \times 10^{5}$ cells into black 96-well Plates. $600 \mu \mathrm{g} / \mathrm{ml} \mathrm{D}$-luciferin was added to each well $5 \mathrm{~min}$ before imaging. Imaging time was $1 \mathrm{~min} /$ plate, Binning $M$. For in vivo imaging, animals were anesthetized with isoflurane and were given D-luciferin $(15 \mathrm{mg} / \mathrm{ml}$ in D-PBS; $100 \mu \mathrm{l} / 10 \mathrm{~g}$ body weight) by intraperitoneal injection. Mice were placed into the light-tight camera box with continuous exposure to isoflurane. Imaging time ranged from $10 \mathrm{~s}$ to $1 \mathrm{~min}$, depending on the tumor model, 5 min after D-luciferin injection. Two mice were imaged each time from a dorsal and a ventral view. The photons emitted from the bioluminescent tumors or cells were detected by the IVIS100 camera system, integrated, digitized and displayed. Numbers of tumors per animal were counted by eye from a dorsal and ventral view with particular regard to the singular count of one metastatic lesion.

\section{Immunohistochemistry}

The following primary antibodies were used: Rabbit polyclonal anti-phospho L-plastin antiserum (phospho L-plastin) and mouse monoclonal anti-L-plastin antibody (LPL4A.1). Isotype- and concentration matched control antibodies (Dako, Hamburg, Germany) served as negative controls.

Immunoenzyme staining was performed on $2-\mu \mathrm{m}$ sections of formalin fixed, paraffin embedded murine organs. Heat induced antigen-retrieval was achieved by incubating the slides in a steam cooker for 5 minutes in citrate buffer, pH 6.1. Slides were further processed using the standard avidin-biotin-complex anti-alkaline phosphatase procedure (Vectorlabs, Burlingame, CA, USA) according to the manufacturer's instructions. The primary antibody was added for 1 hour at $37^{\circ} \mathrm{C}$. A donkey antirabbit biotinylated antibody, 1/100 (Dako), was used as a secondary reagent (30 $\mathrm{min}$ at room temperature). Naphthol AS-biphosphate (Sigma, St. Louis, MO, USA) with New-fuchsin (Merck, Darmstadt, Germany) served as the substrate for alkaline phosphatase.

\section{Statistical methods}

Statistical analyses were performed with GraphPad Prism 4.0. Data are shown as means \pm SEM. Comparisons of means were performed with two-tailed students $t$-test or one-way ANOVA test, respectively. P values were considered significant when $\mathrm{p}<0.05$.

\section{Additional files}

Additional file 1: Figure S1. Bioluminescent activity of tumor cells was not influenced by transfection of the cells. (A) Bioluminescence imaging of PC-3Mpro4/luc (PC3M) cells in vitro. (B) Bioluminescence imaging of $\mathrm{MV} 3$ cells in vitro. The color bar at the right indicates the signal intensity range $\left(\mathrm{p} / \mathrm{s} / \mathrm{cm}^{2} / \mathrm{sr}\right)$.

Additional file 2: Figure S2. S5A-LPL is not phosphorylated in PMA treated MV3 cells as detected by native gel electrophoresis. A) LPL phosphorylation can be detected by native gel electrophoresis followed by Western blotting. LPL is known to be phosphorylated following PMA stimulation of T-cells [18]. This phosphorylation can be visulized by resolving proteins of untreated or PMA-treated T-cells ( $10^{-8} \mathrm{M} ; 30 \mathrm{~min}$ ) on native PAGE and staining of L-plastin on the corresponding Western blot with L-plastin antibodies. In contrast to the situation with control cells, PMA-treatment leads to the occurrence of a second band. This band disappears if lysates from PMA stimulated cells were treated with alkaline phosphatase (AP). B) For an unbiased analysis of LPL phosphorylation in MV3 cells, lysates of control or PMA-treated MV3 cells expressing either wt LPL or S5A LPL were subjected to native gel electrophoresis as described in A. Only wt LPL, but not S5A-LPL showed a band shift after PMA treatment of the cells, which demonstrates that S5A-LPL is not phosphorylated.

\section{Competing interests}

The authors declare that they have no competing interests.

\section{Authors' contributions}

SMR and GHW participated in study design, performing experiments, data analysis and drafting of manuscript. HK, BJ and BS supported in vitro experiments and cloning experiments and provided technical assistance. GVDH and GVDP provided material. FL performed immunhistochemistry experiments. GH and IN were involved in conception of study and manuscript drafting. YS participated in study design, data analysis and writing of the manuscript. All authors read and approved the manuscript.

\section{Acknowledgements}

We thank Jutta Scheuerer for expert technical assistance.

This work was in part funded by the Deutsche Forschungsgemeinschaft (DFG Sa393/3-4 and SFB 938/M to Y.S. and SFB 938/Z2 to F.L.).

\section{Author details}

Institute for Immunology, Ruprecht-Karls-University, Heidelberg, Germany. ${ }^{2}$ Department of Pathology, Ruprecht-Karls-University, Heidelberg, Germany. ${ }^{3}$ Department of Urology, Leiden University Medical Center, Leiden, The Netherlands. ${ }^{4}$ Division of Molecular Immunology, German Cancer Research Center DKFZ, Heidelberg, Germany. ${ }^{5}$ Translational Medicine, Max-Planck Institute for Biochemistry, Martinsried, Germany.

Received: 23 August 2013 Accepted: 8 January 2014 Published: 18 January 2014

\section{References}

1. Gupta GP, Massague J: Cancer metastasis: building a framework. Cell 2006, 127:679-695.

2. Ridley AJ, Schwartz MA, Burridge $K$, Firtel RA, Ginsberg MH, Borisy G, Parsons $J$ T, Horwitz AR: Cell migration: integrating signals from front to back. Science 2003, 302:1704-1709.

3. Valastyan S, Weinberg RA: Tumor metastasis: molecular insights and evolving paradigms. Cell 2011, 147:275-292.

4. Yang ZL, Miao X, Xiong L, Zou Q, Yuan Y, Li J, Liang L, Chen M, Chen S: CFL1 and Arp3 are Biomarkers for Metastasis and Poor Prognosis of Squamous Cell/Adenosquamous Carcinomas and Adenocarcinomas of Gallbladder. Cancer Invest 2013, 31:132-139.

5. Byrne FL, Yang L, Phillips PA, Hansford LM, Fletcher Jl, Ormandy CJ, McCarroll JA, Kavallaris M: RNAi-mediated stathmin suppression reduces lung metastasis in an orthotopic neuroblastoma mouse model. Oncogene 2013. doi:10.1038/onc.2013.11.

6. Stevenson RP, Veltman D, Machesky LM: Actin-bundling proteins in cancer progression at a glance. J Cell Sci 2012, 125:1073-1079. 
7. Vial D, McKeown-Longo PJ: Epidermal growth factor (EGF) regulates alpha5beta1 integrin activation state in human cancer cell lines through the p90RSK-dependent phosphorylation of filamin A. J Biol Chem 2012, 287:40371-40380.

8. Leavitt J, Kakunaga T: Expression of a variant form of actin and additional polypeptide changes following chemical-induced in vitro neoplastic transformation of human fibroblasts. J Biol Dyn 1980, 255:1650-1661.

9. Lin CS, Park T, Chen ZP, Leavitt J: Human plastin genes. Comparative gene structure, chromosome location, and differential expression in normal and neoplastic cells. J Biol Chem 1993, 268:2781-2792.

10. Leavitt J, Chen ZP, Lockwood CJ, Schatz F: Regulation of synthesis of the transformation-induced protein, leukocyte plastin, by ovarian steroid hormones. Cancer Res 1994, 54:3447-3454.

11. Foran E, McWilliam P, Kelleher D, Croke DT, Long A: The leukocyte protein L-plastin induces proliferation, invasion and loss of E-cadherin expression in colon cancer cells. Int J Cancer, 118:2098-2104.

12. Samstag Y, Klemke M: Ectopic expression of L-plastin in human tumor cells: diagnostic and therapeutic implications. Adv Enzyme Regul 2007, 47:118-126.

13. Otsuka M, Kato M, Yoshikawa T, Chen H, Brown EJ, Masuho Y, Omata M, Seki N: Differential expression of the L-plastin gene in human colorectal cancer progression and metastasis. Biochem Biophys Res Commun 2001 289:876-881.

14. Lapillonne A, Coue O, Friederich E, Nicolas A, Del Maestro L, Louvard D, Robine S, Sastre-Garau X: Expression patterns of L-plastin isoform in normal and carcinomatous breast tissues. Anticancer Res 2000, 20:3177-3182

15. Klemke M, Rafael MT, Wabnitz GH, Weschenfelder T, Konstandin MH, Garbi N, Autschbach F, Hartschuh W, Samstag Y: Phosphorylation of ectopically expressed L-plastin enhances invasiveness of human melanoma cells. Int J Cancer 2007, 120:2590-2599.

16. Samstag Y, Eibert SM, Klemke M, Wabnitz GH: Actin cytoskeletal dynamics in T lymphocyte activation and migration. J Leukoc Biol 2003, 73:30-48.

17. Janji B, Giganti A, De Corte V, Catillon M, Bruyneel E, Lentz D, Plastino J, Gettemans J, Friederich E: Phosphorylation on Ser5 increases the F-actin-binding activity of L-plastin and promotes its targeting to sites of actin assembly in cells. J Cell Sci 2006, 119:1947-1960.

18. Wabnitz GH, Kocher T, Lohneis P, Stober C, Konstandin MH, Funk B, Sester $U$, Wilm M, Klemke M, Samstag Y: Costimulation induced phosphorylation of L-plastin facilitates surface transport of the T cell activation molecules CD69 and CD25. Eur J Immunol 2007, 37:649-662.

19. Wabnitz GH, Lohneis P, Kirchgessner H, Jahraus B, Gottwald S, Konstandin M, Klemke M, Samstag Y: Sustained LFA-1 cluster formation in the immune synapse requires the combined activities of L-plastin and calmodulin. Eur J Immunol 2010, 40:2437-2449.

20. De Clercq S, Zwaenepoel O, Martens E, Vandekerckhove J, Guillabert A, Gettemans J: Nanobody-induced perturbation of LFA-1/L-plastin phosphorylation impairs MTOC docking, immune synapse formation and T cell activation. Cell Mol Life Sci 2012, 70:909-22.

21. Pazdrak K, Young TW, Straub C, Stafford S, Kurosky A: Priming of eosinophils by GM-CSF is mediated by protein kinase Cbetallphosphorylated L-plastin. J Immunol 2011, 186:6485-6496.

22. Jones SL, Wang J, Turck CW, Brown EJ: A role for the actin-bundling protein L-plastin in the regulation of leukocyte integrin function. Proc Natl Acad Sci U S A 1998, 95:9331-9336.

23. Wang J, Chen H, Brown EJ: L-plastin peptide activation of alpha(v)beta(3)mediated adhesion requires integrin conformational change and actin filament disassembly. J Biol Chem 2001, 276:14474-14481.

24. Freeley M, O'Dowd F, Paul T, Kashanin D, Davies A, Kelleher D, Long A: L-Plastin Regulates Polarization and Migration in Chemokine-Stimulated Human T Lymphocytes. J Immunol 2012, 188:6357-6370.

25. Shinomiya $\mathrm{H}$ : Plastin family of actin-bundling proteins: its functions in leukocytes, neurons, intestines, and cancer. Int J Biochem Cell Biol 2012, 2012:213492.

26. Delanote V, Vandekerckhove J, Gettemans J: Plastins: versatile modulators of actin organization in (patho)physiological cellular processes. Acta Pharmacol Sin 2005, 26:769-779.

27. Shin SI, Freedman VH, Risser R, Pollack R: Tumorigenicity of virustransformed cells in nude mice is correlated specifically with anchorage independent growth in vitro. Proc Natl Acad Sci U S A 1975, 72:4435-4439.
28. Bos PD, Nguyen DX, Massague J: Modeling metastasis in the mouse. Curr Opin Pharmacol 2010, 10:571-577.

29. Talmadge JE, Fidler IJ: AACR centennial series: the biology of cancer metastasis: historical perspective. Cancer Res 2010, 70:5649-5669.

30. Vollmer TL, Conley FK: Effect of cyclophosphamide on survival of mice and incidence of metastatic tumor following intravenous and intracardial inoculation of tumor cells. Cancer Res 1984, 44:3902-3906.

31. Henning SW, Meuer SC, Samstag Y: Serine phosphorylation of a $67-\mathrm{kDa}$ protein in human $\mathrm{T}$ lymphocytes represents an accessory receptormediated signaling event. J Immunol 1994, 152:4808-4815.

32. Wabnitz GH, Michalke F, Stober C, Kirchgessner H, Jahraus B, van den Boomen DJ, Samstag Y: L-plastin phosphorylation: a novel target for the immunosuppressive drug dexamethasone in primary human T cells. Eur J Immunol 2011, 41:3157-3169.

33. Le Goff E, Vallentin A, Harmand PO, Aldrian-Herrada G, Rebiere B, Roy C, Benyamin Y, Lebart MC: Characterization of L-plastin interaction with beta integrin and its regulation by micro-calpain. Cytoskeleton 2010, 67:286-296

34. Al Tanoury Z, Schaffner-Reckinger E, Halavatyi A, Hoffmann C, Moes M, Hadzic E, Catillon M, Yatskou M, Friederich E: Quantitative kinetic study of the actin-bundling protein L-plastin and of its impact on actin turn-over. Plos One 2010, 5:e9210.

35. Nakahara H, Nomizu M, Akiyama SK, Yamada Y, Yeh Y, Chen WT: A mechanism for regulation of melanoma invasion. Ligation of alpha6beta1 integrin by laminin G peptides. J Biol Chem 1996 271:27221-27224.

36. van den Hoogen C, van der Horst G, Cheung H, Buijs JT, Lippitt JM, Guzman-Ramirez N, Hamdy FC, Eaton CL, Thalmann GN, Cecchini MG, et al: High aldehyde dehydrogenase activity identifies tumor-initiating and metastasis-initiating cells in human prostate cancer. Cancer Res 2010, 70:5163-5173.

37. van der Horst G, van Asten JJ, Figdor A, van den Hoogen C, Cheung $H$, Bevers RF, Pelger RC, van der Pluijm G: Real-time cancer cell tracking by bioluminescence in a preclinical model of human bladder cancer growth and metastasis. Eur Urol 2011, 60:337-343.

\section{doi:10.1186/1476-4598-13-10}

Cite this article as: Riplinger et al:: Metastasis of prostate cancer and melanoma cells in a preclinical in vivo mouse model is enhanced by L-plastin expression and phosphorylation. Molecular Cancer 2014 13:10.

\section{Submit your next manuscript to BioMed Central and take full advantage of:}

- Convenient online submission

- Thorough peer review

- No space constraints or color figure charges

- Immediate publication on acceptance

- Inclusion in PubMed, CAS, Scopus and Google Scholar

- Research which is freely available for redistribution 